\title{
Pre-intervention Imaging
}

National Cancer Institute

\section{Source}

National Cancer Institute. Pre-intervention Imaging. NCI Thesaurus. Code C69195.

Indicates that the imaging procedure was performed immediately prior to a non-surgical intervention. 